\title{
Testosterone, obesity, and waist circumference as determinants of metabolic syndrome in Saudi women
}

This article was published in the following Dove Press journal: Diabetes, Metabolic Syndrome and Obesity:Targets and Therapy

\author{
Sameer H Fatani' \\ Nazik Altayeb Abdelbasit ${ }^{\prime}$ \\ Hiba S Al-Amodi' \\ Maowia Mohamed Mukhtar \\ Abdullatif Taha Babakr' \\ 'Department of Medical Biochemistry, \\ Faculty of Medicine, Umm Al-Qura \\ University, Makkah, Kingdom of Saudi \\ Arabia; ${ }^{2}$ Department of Molecular \\ Biology, Institute of Endemic Diseases, \\ University of Khartoum, Medical \\ Campus, Khartoum, Sudan
}

Background: High serum total testosterone is associated with metabolic syndrome (MS). This study aimed to identify possible alterations in total testosterone and their relationship with plasma glucose, blood pressure, and serum lipid profile.

Methods: One hundred forty-two female subjects were selected to participate in this study, and they were recruited by consultant physicians from the Clinic and Medical Out-Patient, King Abdulaziz Hospital, Kingdom of Saudi Arabia. The anthropometric characteristics were obtained from questionnaires by using standard methods. Blood samples were obtained for the determination of glucose, triglycerides, total cholesterol, low-density lipoprotein, and high-density lipoprotein by using enzymatic methods. Total testosterone was determined by enzyme-linked immunosorbent assay for the quantitative measurement of testosterone in human serum.

Results: Significantly higher concentrations of total testosterone, low-density lipoprotein, and glucose, but lower concentrations of high-density lipoprotein, were observed in subjects with MS compared with women without MS $(P<0.05)$.

Conclusion: This study suggests that high levels of total testosterone and disturbance in lipid profile were associated with MS in Saudi women.

Keywords: obesity, hyperlipidemia, testosterone, metabolic syndrome, lipid profile, androgen, insulin resistance

\section{Background}

Obesity has reached epidemic proportions globally, ${ }^{1-3}$ with more than 1 billion adults being overweight - at least 300 million of them being clinically obese. ${ }^{4}$ It leads to adverse metabolic effects, blood pressure, ${ }^{5}$ and lipid and glucose metabolism mediated by insulin resistance. ${ }^{4}$ This constellation of cardiovascular risk factors constitutes the metabolic syndrome (MS). ${ }^{6} \mathrm{MS}$ is associated with significant increase in cardiovascular morbidity and mortality, ${ }^{7}$ and it is the most common cause of death in the Western world. ${ }^{8}$ Waist circumference (WC) is the best measure of obesity which is combined with other variables to construct a country-specific definition of MS. ${ }^{9}$

In Saudi Arabia, epidemiological studies reported a potential growth in diabetes with a parallel increase in obesity rates. ${ }^{10}$ Currently, $23 \%$ of the health care expenditure along with $17 \%$ of direct medical costs is spent for diabetes. ${ }^{11}$ The components of MS, including central obesity, are particularly common among Saudi population. ${ }^{12}$

The largest study, conducted over a 5-year period between 1995 and 2000 and covering different regions of Saudi Arabia, reported the overall MS prevalence in adults aged between 30 and 70 years to be about $40 \%$, with the most common factor being low level of high-density lipoprotein cholesterol (HDL-C). ${ }^{13}$ The prevalence of cardiovascular
Correspondence: Abdullatif Taha Babak Department of Medical Biochemistry, Faculty of Medicine, Umm Al-Qura University, PO Box 7607 Abdia, Makkah 21 955, Kingdom of Saudi Arabia Tel +966 I2 25270000 extn 4322 Fax +966 I2 25270000 extn 4319 Email atbabakr@uqu.edu.sa 
disease (CVD) risk factors is high among women in Saudi Arabia especially in cases of obesity and physical inactivity. ${ }^{11,14}$ Public health authorities must implement solutions from a gender-specific aspect to reverse the trend and decrease the prevalence of CVDs among Saudi women. ${ }^{14}$

The first study of MS in Saudi Arabia was published in $2002 .{ }^{12}$ Two other smaller studies conducted in the central region of Saudi Arabia reported the prevalence to be $31.4 \%$ in men $^{15}$ and $35.3 \%$ in the whole population. ${ }^{16}$ Another study, conducted recently in the city of Jeddah in the western region, reported the MS prevalence to be $21 \%$ with low HDL-C as a common component. ${ }^{13}$ The present study was conducted to evaluate the levels of testosterone, obesity, and WC as determinants of MS in Saudi women.

Testosterone is a hormone that plays a key role in the metabolism of carbohydrate, fat, and protein. With a major influence on body fat composition in men, testosterone deficiency was suggested to be associated with reduced insulin sensitivity, impaired glucose tolerance, elevated triglycerides (TGs) and cholesterol, and low HDL-C in previous studies. ${ }^{17}$ All these factors are found in the MS and type 2 diabetes. Numerous studies showed associations between serum testosterone and insulin resistance or MS and type 2 diabetes risk in women; however, the conclusion about causal relationship still needs more studies. ${ }^{18}$ The present study was aimed to address testosterone levels with the components of MS in Saudi women.

\section{Subjects and methods Ethical approval}

This retrospective study was conducted in the Biochemistry Laboratory, Department of Medical Laboratories, Faculty of Medicine, Umm Al-Qura University, Kingdom of Saudi Arabia from January 2015 to March 2017. Written informed consent was obtained from all participants. The overall study methodology was approved by the Biomedical Ethics Committee, Faculty of Medicine, Umm Al-Qura University, Mecca Al-Mukarama, Kingdom of Saudi Arabia.

\section{Subjects}

A total of 142 female subjects aged between 20 and 60 years were recruited for this study. Of them, 95 subjects with high WC $(\geq 88 \mathrm{~cm})$ and body mass index (BMI $\left.\geq 30 \mathrm{~kg} / \mathrm{m}^{2}\right)$ and 47 apparently healthy women in normal weight as controls (BMI $\leq 25 \mathrm{~kg} / \mathrm{m}^{2}$ ) were selected. Those on lipid-lowering drugs and hormonal medications, with CVDs like stroke, and who did not give consent were excluded. Information of age, maternity, menarche, and menstrual cycle disturbance were obtained using a questionnaire. Weight, height, WC, and systolic and diastolic blood pressures (SBP and DBP, mean of three consecutive measurements) were measured.

\section{Sampling}

Following the overnight fasting period, $5 \mathrm{~mL}$ of whole venous blood samples were withdrawn from each subject. The sample was divided into two parts (plain and EDTA tubes) as serum and plasma samples, respectively. The samples were used for the estimation of plasma, fasting blood glucose, and total testosterone. Serum was used for the estimation of TGs, total cholesterol (TC), low- and high-density lipoproteins (LDL and HDL), and total testosterone.

\section{Metabolic syndrome}

The subjects were recruited by consultant physicians from the Clinic and Medical Out-Patient, King Abdulaziz Hospital. MS is defined by using National Cholesterol Education Program Adult Treatment Panel III guidelines, and it was diagnosed on the basis of five major components: 1) hypertriglyceridemia $\mathrm{TG} \geq 150 \mathrm{mg} / \mathrm{dL}$; 2) $\mathrm{WC} \geq 102 \mathrm{~cm}$ for men and $\geq 88 \mathrm{~cm}$ for women; 3) $\mathrm{SBP} \geq 130 \mathrm{mmHg}$, DBP $\geq 85 \mathrm{mmHg}$; 4) HDL-C $\leq 50$; and 5) fasting plasma glucose $\geq 110 \mathrm{mg} / \mathrm{dL} .{ }^{19}$

\section{Biochemical investigation}

Serum TC, TGs, HDL-C, and LDL-C were estimated by using direct homogenous method and enzymatic colorimetric test. The kits were provided by HUMAN Gesellschaftfür Biochemica und Diagnostica mbH.

Fasting plasma glucose concentration was determined with an automated analyzer (HumaStar 80) by using glucose oxidase-phenol and 4-aminoantipyrine (GOD-PAP) method and an enzymatic colorimetric test kit. The kits were provided by HUMAN Gesellschaftfür Biochemica und DiagnosticambH (REF 10720p).

\section{Hormone assays}

The serum level of total testosterone was determined by using the enzyme-linked immunosorbent assay (ELISA) test for the quantitative determination of testosterone in human serum. The kits were provided by HUMAN Gesellschaftfür Biochemica und Diagnostica mbH (REF 55010, 96 tests), and the test was performed according to the manufacturer's instructions and with the lowest detection limit of $0.06 \mathrm{ng} /$ $\mathrm{mL}$ and a coefficient of variation $\leq 10 \%$.

\section{Blood pressure}

Blood pressure was measured by a Microlife blood pressure monitor (Microlife A200 AFIB, Taipei, Taiwan). Two values, 
the systolic (upper) value and the diastolic (lower) value, are always measured. Any activity was avoided, and the woman was made to sit down for at least 5 minutes before measurement and to relax; close-fitting garments from the upper arm was removed, shirt sleeves were not rolled up, and the cuff was fitted closely and positioned $2 \mathrm{~cm}$ above the elbow. The cuff pumped up automatically. When the correct pressure was reached, the pumping was stopped and the pressure fell gradually; the cuff was removed after the measurement. Hypertension was defined as SBP $\geq 130 \mathrm{mmHg}$ and DBP $\geq 85 \mathrm{mmHg}$.

\section{Statistical analysis}

All statistical analysis was performed using IBM SPSS software version 16.0 (SPSS Inc., Chicago, IL, USA). Descriptive measures were done for each variable in every group. Data in the form of continuous variables were presented as mean \pm SD. Pearson correlation coefficient was used to determine the relationship between variables. Since the test shows that the studied variables are normally distributed for comparing the quantitative variables in the two studied groups, independent sample $t$-test was used. $P<0.05$ was considered as statistically significant. All results are two-tailed. GraphPad Prism 7 software version 7.03 (GraphPad Software, Inc., La Jolla, CA, USA) was used for graphing.

\section{Results}

In this study, we included 142 Saudi women aged between 20 and 60 years. They were divided as cases of MS and controls. Blood pressure, blood glucose, lipid profile, and serum testosterone were measured along with the anthropometric data.

Table 1 provides the age, BMI, WC, blood pressure, and gynecologic data. It has been found that the mean age of the cases was 43.49 years and that of the controls was 39.49 years. The BMI and WC of women with MS were found to be 41.9 and $107.5 \mathrm{~cm}$, respectively. Mean DBP was reported as 134 and $113 \mathrm{~mm} / \mathrm{Hg}$ among case and controls, respectively.

Table 2 and Figures 1 and 2 show the estimation of blood glucose and lipid profile. The mean fasting blood glucose was found to be $163.2 \mathrm{mg} / \mathrm{dL}$ among women with MS and $66.11 \mathrm{mg} / \mathrm{dL}$ among controls. The lipid profile was reported to be as follows: the mean TGs, TC, LDL, and HDL were $194.2,286.3,198.0$, and $53.73 \mathrm{mg} / \mathrm{dL}$ among cases and 68.23, $113.9,106.1$, and $98.49 \mathrm{mg} / \mathrm{dL}$ for controls, respectively.

Correlation between lipids profile and BMI among the whole study group (cases and controls) is shown in Figure 2A-D, reflecting that a strong positive correlation exists between BMI and blood glucose, serum TGs, cholesterol, and LDL. A negative correlation was reported between BMI and serum HDL as shown in Figure 3.

Correlation between glucose and lipid profile with WC among the whole study group (cases and controls) is shown in

Table 2 Blood glucose and lipid profile among study groups

\begin{tabular}{llll}
\hline Index $(\mathbf{m g} / \mathbf{d L})$ & Control $\mathbf{n = 4 7}$ & MS $\mathbf{n = 9 5}$ & P-value \\
\hline Glucose & $66.1 \mathrm{I} \pm 3.66$ & $163.2 \pm 6.13$ & $<0.0 \mathrm{I}$ \\
Triglyceride & $68.23 \pm 4.534$ & $194.2 \pm 1 \mathrm{I} .6 \mathrm{I}$ & $<0.000 \mathrm{I}$ \\
Cholesterol & $113.9 \pm 6.347$ & $286.31 \pm 13.9$ & $<0.000 \mathrm{I}$ \\
LDL-C & $106.1 \pm 6.86$ & $198.0 \pm 9.4$ & $<0.000 \mathrm{I}$ \\
HDL-C & $98.49 \pm 4.77$ & $53.73 \pm 9.82$ & $<0.000 \mathrm{I}$ \\
\hline
\end{tabular}

Note: Values are expressed as mean \pm SD

Abbreviations: LDL-C, low-density lipoprotein cholesterol; HDL-C, high-density lipoprotein cholesterol; MS, metabolic syndrome.

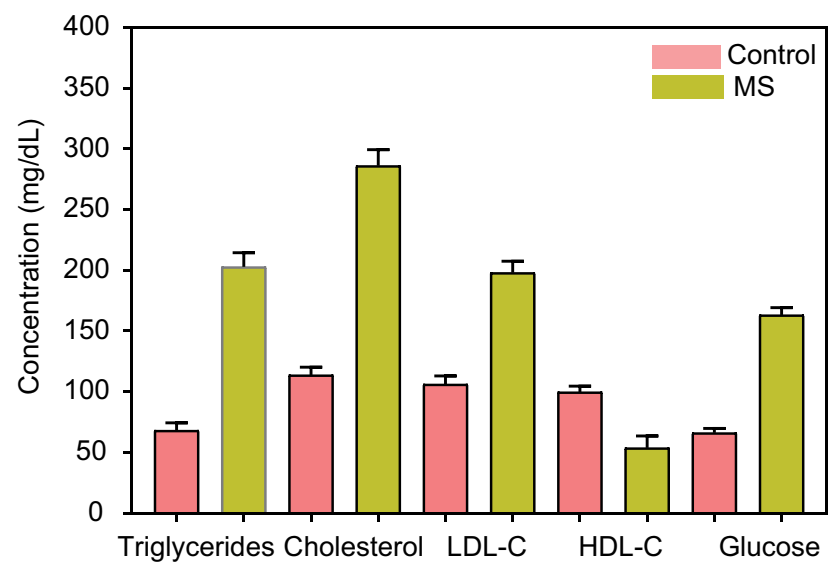

Figure I Glucose and lipid profile in controls and cases of MS.

Abbreviations: MS, metabolic syndrome; LDL-C, low-density lipoprotein cholesterol; HDL-C, high-density lipoprotein cholesterol.

Table I Anthropometric data among study female groups

\begin{tabular}{|c|c|c|c|}
\hline Index & Controls $n=47$ & MS $n=95$ & P-value \\
\hline Age (years) & $39.49 \pm 10.23$ & $43.49 \pm 9.416$ & $<0.05$ \\
\hline Body mass index $\left(\mathrm{kg} / \mathrm{m}^{2}\right)$ & $24.53 \pm 2.73$ & $41.905 \pm 6.08$ & $<0.001$ \\
\hline Waist circumference $(\mathrm{cm})$ & $73.81 \pm 13.93$ & $107.46 \pm 12.06$ & $<0.001$ \\
\hline Systolic blood pressure $(\mathrm{mmHg})$ & $70.19 \pm 3.66$ & $106.62 \pm 5.67$ & $<0.001$ \\
\hline Diastolic blood pressure (mmHg) & $113.27 \pm 1.17$ & $134.58 \pm 2.08$ & $<0.001$ \\
\hline
\end{tabular}

Note: Values are expressed as mean \pm SD

Abbreviation: MS, metabolic syndrome. 
A

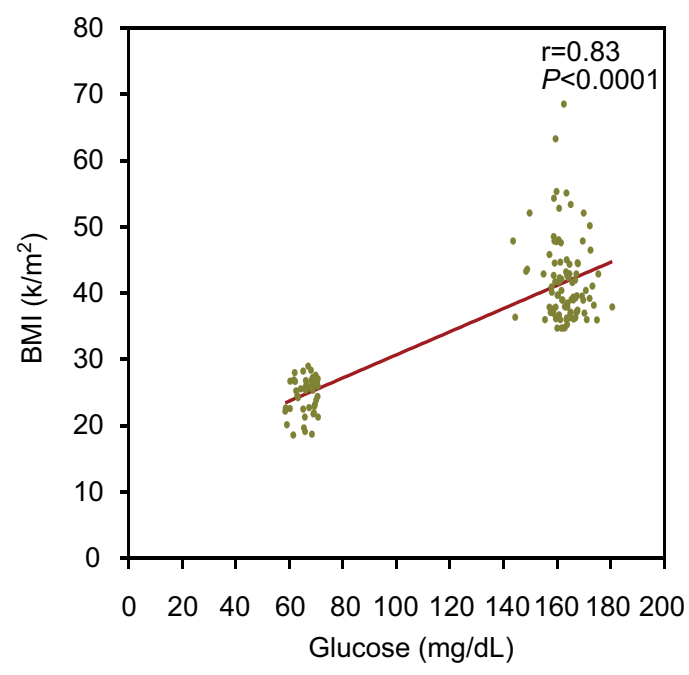

C



B

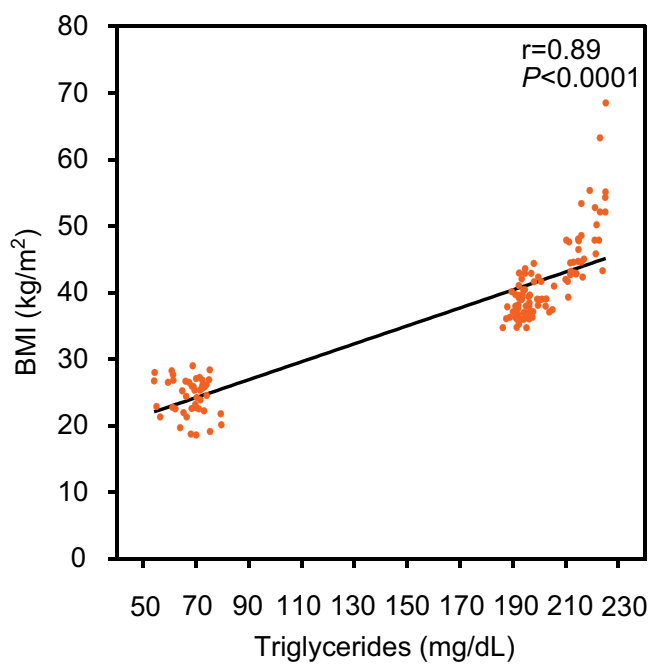

D

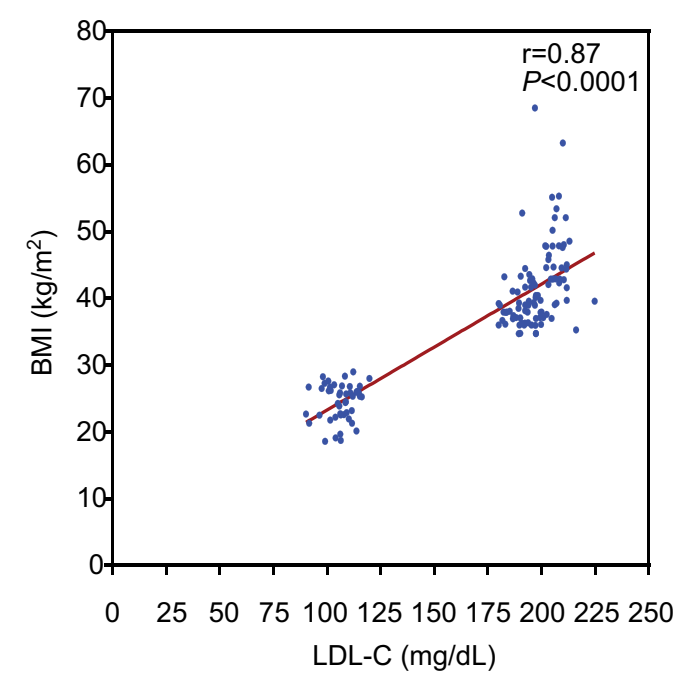

Figure 2 Correlation between glucose (A), triglycerides (B), cholesterol (C), LDL-C (D), and BMI among the whole study group. Abbreviations: LDL-C, low-density lipoprotein cholesterol; BMI, body mass index.

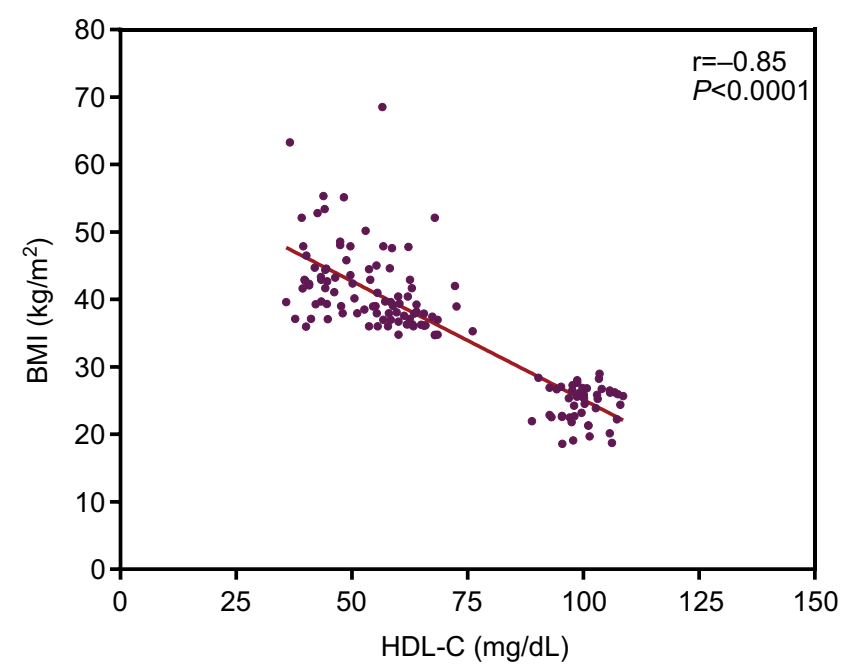

Figure 3 Correlation between HDL-C and BMI among the whole study group. Abbreviations: HDL-C, high-density lipoprotein cholesterol; BMI, body mass index.
Figure 4A-D, reflecting a strong positive correlation between WC and blood glucose, serum TGs, cholesterol, and LDL. A negative correlation was reported between WC and serum HDL as shown in Figure 5.

The levels of testosterone were also found to be higher in women with MS than in women without MS as shown inv Figure 6.

\section{Discussion}

This study aimed to detect the relationship between obesity, BMI, and WC as determinants of MS among Saudi women. The age of the study group ranged between 20 and 60 years. The main findings of the current study showed a strong significant relationship between BMI and WC with MS. The mean age was higher in women with MS compared to controls (43 and 39 years, respectively). 
A

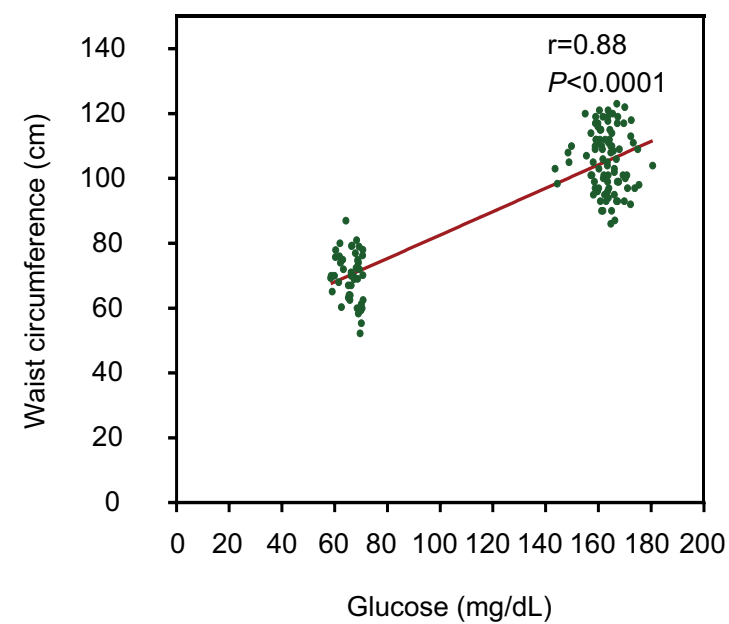

C

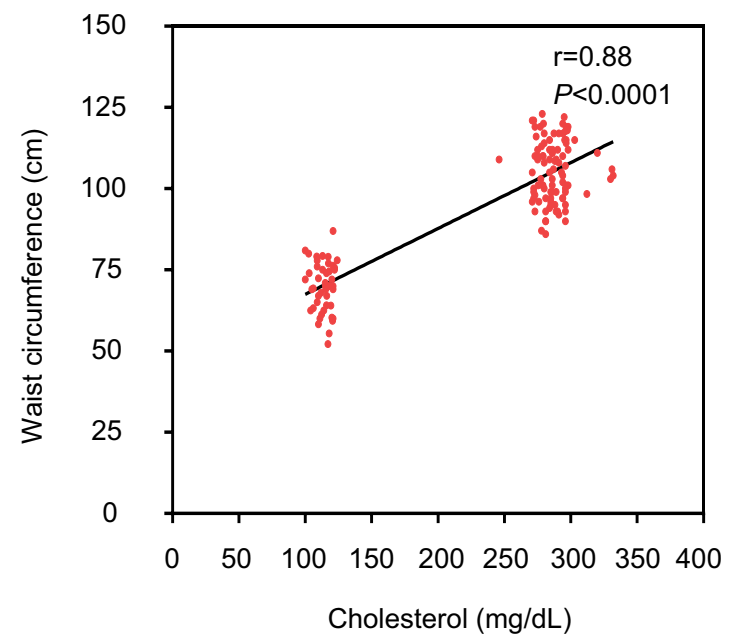

B

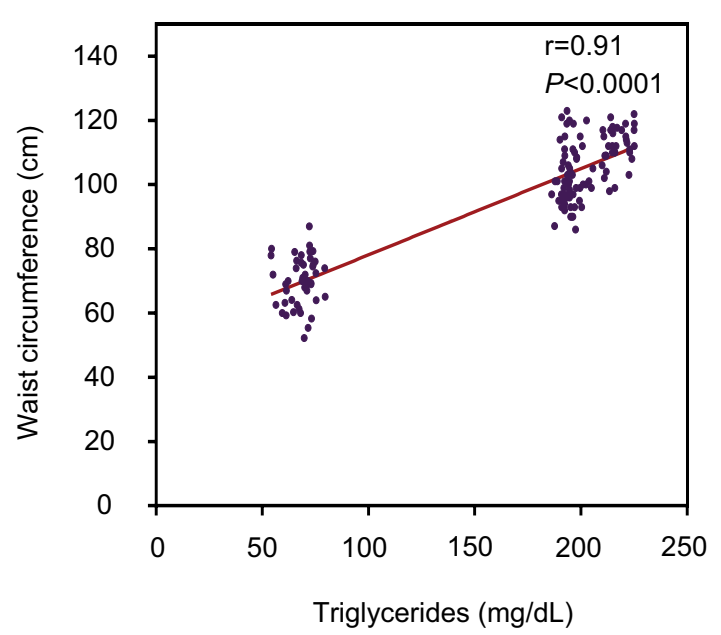

D

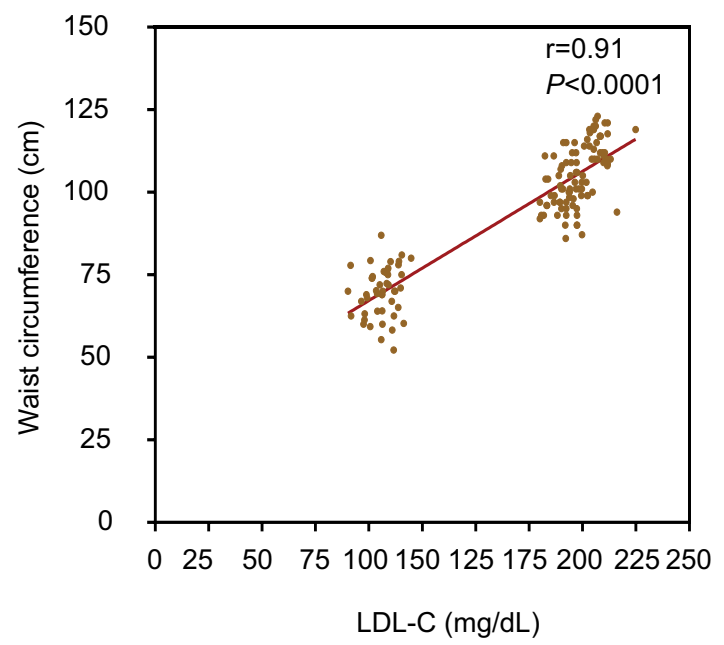

Figure 4 Correlation between glucose, cholesterol, triglycerides, LDL-C, and waist circumference among the whole study group. (A) Fasting blood sugar levels and BMI; (B) triglycerides and BMI; (C) cholesterol and BMI; (D) LDL-C and BMI.

Abbreviation: LDL-C, low-density lipoprotein cholesterol.

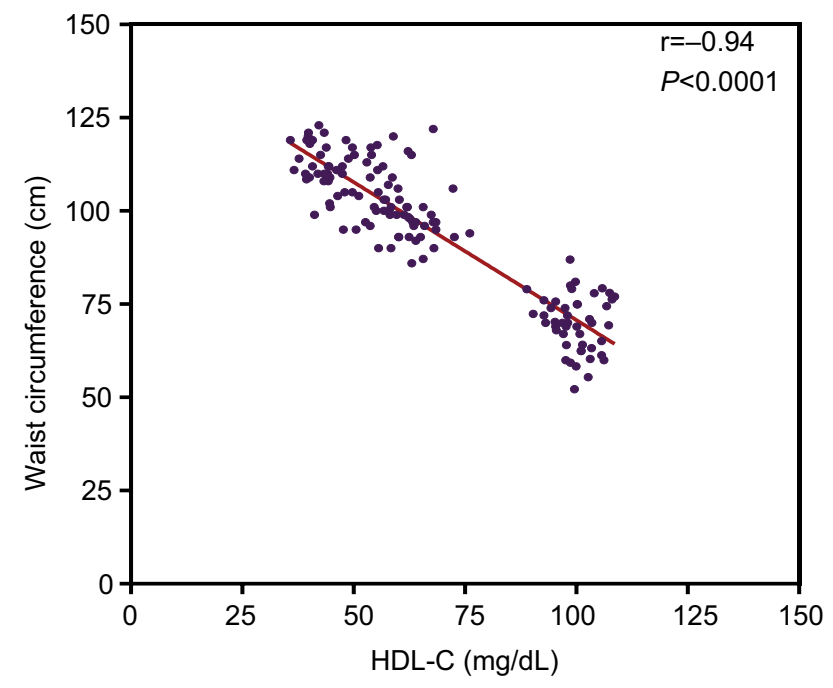

Figure 5 Correlation between HDL-C and waist circumference among the whole study group.

Abbreviation: HDL-C, high-density lipoprotein cholesterol.
The fact that an increase in TG levels is observed along with the increment of the WC and BMI is similar to the findings in a previous study. ${ }^{20}$ The study conducted by $\mathrm{Du}$ et $\mathrm{al}^{21}$ has demonstrated that the HDL-C level is affected by weight. Furthermore, in this study, the average of the HDL-C levels in normal-weight and obese women with MS is higher in comparison with our results. ${ }^{21}$ Another finding of our study that should be emphasized is the fact that the HDL-C level decreases along with the increase of total testosterone concentration. There was a significant change in the LDL-C level parallel to the increment of WC, BMI, and testosterone level.

With regard to the effect on metabolic abnormal values which were in our study for TC, HDL-C, and LDL-C, similar strong association was found between their abnormal levels and abnormal total testosterone concentration, for high TGs 


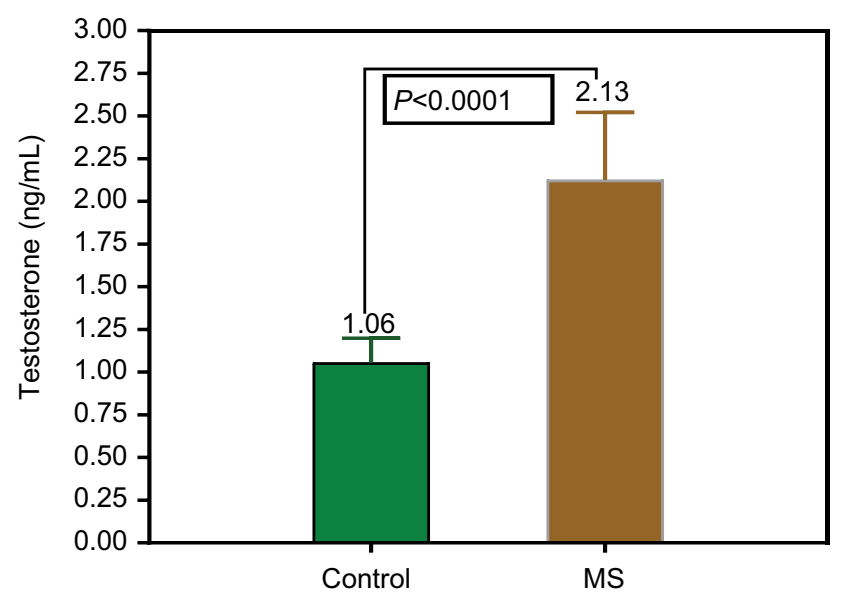

Figure 6 The levels of testosterone in controls and women with MS. Abbreviation: MS, metabolic syndrome.

$(P=0.0001)$, TC $(P=0.0001)$, LDL-C $(P=0.0001)$, and low levels of HDL $(P=0.0001)$.

With regard to hormonal effects, significant difference between the fasting blood levels of testosterone $(P=0.0001)$ was found between the two groups. Higher levels of testosterone in women with MS was suggested in previous studies. ${ }^{18}$ Excess of androgen could potentially contribute to the pathogenesis of insulin resistance and MS in women with polycystic ovary syndrome. ${ }^{18}$ The data in the present study show that women with MS have twofold increase in testosterone levels. The causal relationship needs more investigation and larger studies.

Recently, obesity is reaching epidemic proportions with worldwide figures estimated at 1.4 billion and rising every year. ${ }^{22}$ Obesity affects all socioeconomic backgrounds and different ethnicities and is considered as a prerequisite for MS. MS is a clustering of risk factors that increase the risk of type 2 diabetes mellitus and CVDs. The analysis of the distribution of MS suggested that what follows under the common definition of this pathologic condition is not a unique entity, it is rather a constellation of cluster of components that may differ across different countries. ${ }^{23}$ As these pathologic conditions are among the leading causes of deaths worldwide, it is of critical importance to continuously evaluate the factors associated with increased prevalence of MS especially in communities of modern and sedentary lifestyles. Further studies are recommended to validate the risk factors and biochemical parameters associated with the incidence of MS.

\section{Conclusion}

In summary, the data of this study showed a significant positive correlation between BMI, WC, and disturbance in lipid profile and MS, and a high total testosterone concentrations was found in Saudi women with MS.

\section{Acknowledgment}

The authors thank the Institute of Scientific Research and Revival of Islamic Culture, Umm Al-Qura University, Makkah, Kingdom of Saudi Arabia, for funding this project.

\section{Author contributions}

SHF contributed to design of the study, interpretation of biochemical laboratory investigations, and editing, styling, writing, and revising of the main manuscript text. HSA and MMM were involved in design of the study, interpretation of biochemical laboratory investigations, preparation of tables and figures, and editing, styling, writing, and revising of the main manuscript text. NAA and ATB contributed to biochemical laboratory investigations, statistical analysis, preparation of tables and figures, and editing, styling, writing, and revising of the main manuscript text. All the authors read and approved the final manuscript.

\section{Disclosure}

The authors report no conflicts of interest in this work.

\section{References}

1. Alnohair S. Obesity in Gulf countries. Int J Health Sci (Qassim). 2014;8(1):79-83.

2. Zadjali F, Al-Bulushi A, Al Hassani F, Al Hinai M. Proportion of night eating syndrome in Arab population of Oman. $J$ Eat Disord. 2015;3(1):43.

3. Fouad M, Ismail MI, Gaballah A, Reyad E, Eldeeb S. Prevalence of obesity and risk of chronic kidney disease among young adults in Egypt. Indian J Nephrol. 2016;26(6):413.

4. Goyal M, Chandola H, Goyal R. A clinical study on the role of Agnimanthadi compound and Vashpa Svedana in the management of Sthaulya (obesity). Ayu. 2013;34(4):390.

5. Orces $\mathrm{CH}$, Gavilanez EL. The prevalence of metabolic syndrome among older adults in Ecuador: results of the SABE survey. Diabetes Metab Syndr Clin Res Rev. Epub 2017 Apr 11.

6. Braunschweig CL, Gomez S, Sheean P, Tomey KM, Rimmer J, Heller T. Nutritional status and risk factors for chronic disease in urbandwelling adults with down syndrome. Am J Ment Retard. 2004; 109(2): 186 .

7. Girisha BS. Metabolic syndrome in psoriasis among urban South Indians: a case control study using SAM-NCEP criteria. J Clin Diagn Res. Epub 2017 Feb 1.

8. Ranasinghe P, Mathangasinghe Y, Jayawardena R, Hills AP, Misra A. Prevalence and trends of metabolic syndrome among adults in the Asia-Pacific region: a systematic review. BMC Public Health. 2017; 17(1):101

9. Al-Thani MH, Al-Thani AAM, Cheema S, et al. Prevalence and determinants of metabolic syndrome in Qatar: results from a National Health Survey. BMJ Open. 2016;6(9):e009514.

10. Elhadd T, Al-Amoudi A, Alzahrani A. Epidemiology, clinical and complications profile of diabetes in Saudi Arabia: a review. Ann Saudi Med. 2007;27(4):241. 
11. Ahmed HG, Ginawi IA, Elasbali AM, Ashankyty IM, Al-hazimi AM. Prevalence of obesity in Hail Region, KSA: in a comprehensive survey. J Obes. 2014;2014:1-5.

12. Abo Gazalah F, Al Reshidi F. The prevalence of metabolic syndrome among chronic disease patients in Alwazarat health center at Prince Sultan Military Medical City, Riyadh, Saudi Arabia, 2014. Int J Med Sci Public Health. 2016;5(1):125-134.

13. Bahijri SM, Al Raddadi RM. The importance of local criteria in the diagnosis of metabolic syndrome in Saudi Arabia. Ther Adv Endocrinol Metab. 2013;4(2):51-59.

14. Alshaikh MK, Filippidis FT, Baldove JP, Majeed A, Rawaf S. Women in Saudi Arabia and the prevalence of cardiovascular risk factors: a systematic review. J Environ Public Health. 2016;2016:1-15.

15. Al-Daghri NM, Al-Attas OS, Alokail MS, Alkharfy KM, Sabico SLB, Chrousos GP. Decreasing prevalence of the full metabolic syndrome but a persistently high prevalence of dyslipidemia among adult Arabs. PLoS One. 2010;5(8):e12159.

16. Alzahrani AM, Karawagh AM, Alshahrani FM, Naser TA, Ahmed AA, Alsharef EH. Prevalence and predictors of metabolic syndrome among healthy Saudi adults. Br J Diabetes Vasc Dis. 2012;12(2):78-80.
17. Kelly DM, Jones TH. Testosterone: a metabolic hormone in health and disease. J Endocrinol. 2013;217(3):R25-R45.

18. Corbould A. Effects of androgens on insulin action in women: is androgen excess a component of female metabolic syndrome? Diabetes Metab Res Rev. 2008;24(7):520-532.

19. Patel SM, Ratcliffe SJ, Reilly MP, et al. Higher serum testosterone concentration in older women is associated with insulin resistance, metabolic syndrome, and cardiovascular disease. J Clin Endocrinol Metab. 2009;94(12):4776-4784.

20. Özkaya İ, Bavunoglu I, Tunçkale A. Body mass index and waist circumference affect lipid parameters negatively in Turkish women. Am J Public Health Res. 2014;2(6):226-231.

21. Du S-M, Ma G-S, Li Y-P, et al. Relationship of body mass index, waist circumference and cardiovascular risk factors in Chinese adult. Biomed Environ Sci. 2010;23(2):92-101.

22. Timper K, Bruning JC. Hypothalamic circuits regulating appetite and energy homeostasis: pathways to obesity. Dis Model Mech. 2017;10(6):679-689.

23. Scuteri A, Laurent S, Cucca F, et al. Metabolic syndrome across Europe: different clusters of risk factors. Eur J Prev Cardiol. 2015;22(4):486-491.
Diabetes, Metabolic Syndrome and Obesity: Targets and Therapy is an international, peer-reviewed open-access journal committed to the rapid publication of the latest laboratory and clinical findings in the fields of diabetes, metabolic syndrome and obesity research. Original research, review, case reports, hypothesis formation, expert opinion and commentaries are all considered for publication. The manuscript management system is completely online and includes a very quick and fair peer-review system, which is all easy to use. Visit http://www.dovepress.com/testimonials.php to read real quotes from published authors.

Submit your manuscript here: https://www.dovepress.com/diabetes-metabolic-syndrome-and-obesity-targets-and-therapy-journal 